\title{
SYMPLECTIC ASPECTS OF THE TT*_TODA EQUATIONS
}

\author{
RYOSUKE ODOI
}

\begin{abstract}
We evaluate explicitly, in terms of the asymptotic data, the ratio of the constant pre-factors in the large and small $x$ asymptotics of the tau functions for global solutions of the $\mathrm{tt}^{*}$-Toda equations. This constant problem for the sinh-Gordon equation, which is the case $n=1$ of the $\mathrm{tt}^{*}$-Toda equations, was solved by C. A. Tracy [18. We also introduce natural symplectic structures on the space of asymptotic data and on the space of monodromy data for a wider class of solutions, and show that these symplectic structures are preserved by the Riemann-Hilbert correspondence.
\end{abstract}

\section{Contents}

1. Introduction

2. The $\mathrm{tt}^{*}$-Toda equations

3. The Hamiltonian formulation

4. Symplectic structures and the Riemann-Hilbert correspondence

5. Tau functions and the constant problem

Appendix A. Monodromy data

References

\section{INTRODUCTION}

Painlevé equations may be formulated as Hamiltonian systems. This has led to an important role in the theory of such equations for concepts from classical mechanics and symplectic geometry, such as canonical coordinates, tau-functions, and moduli spaces of solutions with symplectic structures. The benefit of the symplectic point of view is that it illuminates a path to the study of more general nonlinear differential equations, especially those which are "integrable".

The Painlevé equations themselves are scalar ordinary differential equations of second order, and this facilitates explicit calculations. For systems, or higher-order equations, geometry plays a more essential role.

Indeed, a considerable amount of general theory has been developed, for example by Hitchin [8], Boalch [1], building on earlier work of Schlesinger [17], Jimbo-MiwaUeno [12]. On the other hand examples are rather scarce, partly because of the difficulty of carrying out explicit calculations, and partly because of the lack of interesting concrete example for higher rank systems.

The purpose of this article is to explain some symplectic aspects of the $\mathrm{tt}^{*}$ Toda equations, a system of nonlinear ordinary differential equation of "Painlevé type", which is a relatively recent example arising in physics. The tt* equations

Key words and phrases. tt* equations, $\tau$-function, Riemann-Hilbert correspondence. 
(topological-anti topological fusion equations) arose in the work of Cecotti and Vafa on supersymmetric quantum field theory $([2],[3])$, and the $t^{*}$-Toda equations are a special case of these equations of "Toda type". The simplest nontrivial case of the $\mathrm{tt}^{*}$-Toda equations is the (radial) sinh-Gordon equation, which is in fact a case of the third Painlevé equation. It was investigated - for similar physical reasons - by McCoy-Tracy-Wu [13], and their work had far-reaching consequences.

More recently, the $t^{*}$-Toda equations were investigated in detail by Guest-ItsLin [6, 7] and by Mochizuki [14, 15], and our motivation was to put some of these results into a symplectic context and investigate them further. We have succeeded to do this only for a certain subset of solutions - an open subset of the moduli space of all solutions - but the results are encouraging, and have already led to a new application, which we shall explain later.

The paper is organized as follows. After a brief review of the $t^{*}$-Toda equations in section 2, we give their Hamiltonian formulation in section 3. In section 4 we explain the symplectic structures on the space of solutions that we consider and on a corresponding space of monodromy data. The correspondence between solutions (asymptotic data) and monodromy data (Stokes matrices and connection matrices) is an example of the Riemann-Hilbert correspondence for meromorphic connections with irregular singularities. Our first main result (Theorem 4) is that this correspondence preserves the symplectic structures. This is consistent with the general results of Boalch [1, but we shall go further and give an explicit generating function which relates the corresponding canonical coordinates (Theorem 7 ).

In section 5 we give an application of these results to the asymptotics of the tau function. For each solution of the tt*-Toda equation there is a corresponding tau function, and it is the properties these tau functions (rather than the solutions themselves) which are important for many applications in physics.

A recent example is the work of Its, Lisovyy, and Tykhyy [10, in which the structure of the tau functions was elucidated using representation theory (conformal blocks), as a consequence of AGT duality in physics. This was used to solve the "constant problem" for Painlevé equations, i.e. the problem of finding the constant which relates the short-distance and long-distance expansions of the tau function. In the case of the (radial) sine-Gordon equation, a rigorous and more direct proof was given by Its and Prokhorov [11. Our second main result makes use of their method in order to solve this "constant problem" for the tt*-Toda equations. As in [11, we find that the (explicit) generating function plays a crucial role (Theorem 111).

This work is part of the author's Ph. D. thesis at Waseda University. He would like to acknowledge his supervisor, Prof. Martin Guest for his support throughout this work and his guidance in this field. He would like to acknowledge Prof. Alexander Its for his friendly advice and his suggestions regarding the constant problem. He is also glad to acknowledge the financial support from the Mathematics and Physics Unit "Multiscale Analysis, Modelling and Simulation", Top Global University Project, Waseda University.

\section{The TT*-TODA EQUATIONS}

Let a positive integer $n$ be fixed. The $\mathrm{tt}^{*}$-Toda equations are

$$
2\left(w_{i}\right)_{t \bar{t}}=-e^{2\left(w_{i+1}-w_{i}\right)}+e^{2\left(w_{i}-w_{i-1}\right)}, w_{i}: \mathbb{C}^{*} \rightarrow \mathbb{R}, i \in \mathbb{Z},
$$


where, for all i, $w_{i}=w_{i+n+1}, w_{i}=w_{i}(|t|)\left(t \in \mathbb{C}^{*}\right)$, and

$$
w_{0}+w_{n}=0, w_{1}+w_{n-1}=0, \ldots \text { (anti-symmetry condition). }
$$

The equations (2.1) are equivalent to the flatness of $\nabla:=d+\alpha$, i.e. the zero curvature equation $d \alpha+\alpha \wedge \alpha=0$, where

$$
\begin{gathered}
\alpha:=\left(w_{t}+\frac{1}{\lambda} W^{T}\right) d t+\left(-w_{\bar{t}}+\lambda W\right) d \bar{t} \\
w=\left(\begin{array}{ccc}
w_{0} & & \\
& \ddots & \\
& & w_{n}
\end{array}\right), W=\left(\begin{array}{ccc}
0 & e^{w_{1}-w_{0}} & \\
0 & \ddots & \\
0 & \ddots & e^{w_{n}-w_{n-1}} \\
& & 0
\end{array}\right) .
\end{gathered}
$$

The $t^{*}$-Toda equations are also equivalent to the isomonodromy condition for the following ordinary differential equation

$$
\frac{d \Psi}{d \zeta}=\left(-\frac{1}{\zeta^{2}} W-\frac{1}{\zeta} x w_{x}+x^{2} W^{T}\right) \Psi,
$$

where $x:=|t|$.

Generically, the local solutions near $x=0$ of the $\mathrm{tt}^{*}$-Toda equations are parametrized by real numbers $\gamma_{i}, \rho_{i}$ as follows [7, 14, 15]:

$$
2 w_{i}(x)=\gamma_{i} \log x+\rho_{i}+o(1) \quad \text { as } x \rightarrow 0 .
$$

We call the parameters $\gamma_{i}, \rho_{i}$ the asymptotic data. "Generically" means $-2<$ $\gamma_{i+1}-\gamma_{i}<2$; the general case has $-2 \leq \gamma_{i+1}-\gamma_{i} \leq 2$. We assume the generic condition from now on.

There is another important set of data $m_{i}, e_{i}^{\mathbb{R}}$ called the monodromy data. These are eigenvalues of certain matrices $M$ and $E$, which are related to monodromy data such as Stokes matrices. See [7] or the appendix for details. The proof in [7] is for the case $n=3$, but exactly the same method provides the results of Theorem 1 and 2 below for general $n$.

Theorem 1. 7] The monodromy data $m_{i}, e_{i}^{\mathbb{R}}$ may be expressed in terms of the asymptotic data as follows:

$$
\begin{aligned}
m_{i} & =-\frac{1}{2} \gamma_{i} \\
e_{i}^{\mathbb{R}} & = \begin{cases}e^{\rho_{i}} 2^{2 \gamma_{i}} \frac{X_{n-i}\left(\gamma_{0}, \ldots, \gamma_{(n-1) / 2},-\gamma_{(n-1) / 2}, \ldots,-\gamma_{0}\right)}{X_{i}\left(\gamma_{0}, \ldots, \gamma_{(n-1) / 2},-\gamma_{(n-1) / 2}, \ldots,-\gamma_{0}\right)} & n: \text { odd } \\
e^{\rho_{i}} 2^{2 \gamma_{i}} \frac{X_{n-i}\left(\gamma_{0}, \ldots, \gamma_{(n-2) / 2}, 0,-\gamma_{(n-2) / 2}, \ldots,-\gamma_{0}\right)}{X_{i}\left(\gamma_{0}, \ldots, \gamma_{(n-2) / 2}, 0,-\gamma_{(n-2) / 2}, \ldots,-\gamma_{0}\right)} & n: \text { even }\end{cases}
\end{aligned}
$$

where

$$
X_{k}\left(\gamma_{0}, \ldots, \gamma_{n}\right):=\prod_{j=1}^{n} \Gamma\left(\frac{\gamma_{k}-\gamma_{k+j}+2 j}{2(n+1)}\right)\left(\gamma_{j+n+1}=\gamma_{j}\right)
$$

Global solutions can be parametrized only by the $\gamma_{i}$ (or only by the $m_{i}$ ), that is, for global solutions the $\rho_{i}$ are determined by the $\gamma_{i}$ :

Theorem 2. 7] For global solutions (i.e. solutions which are smooth for $0<x<$ $\infty)$ we have

i.e. $e_{i}^{\mathbb{R}}=1$.

$$
\rho_{i}=-(2 \log 2) \gamma_{i}+\log \left(X_{i} / X_{n-i}\right),
$$




\section{The Hamiltonian formulation}

Next, we introduce a Hamiltonian function and a symplectic form.

Let $\lfloor x\rfloor:=\max \{n \in \mathbb{Z}: n \leq x\}$ for $x \in \mathbb{R}$. The tt*-Toda equations can be written as a non-autonomous Hamiltonian system,

$$
\begin{aligned}
& \left(w_{i}\right)_{x}=\frac{\partial H}{\partial \tilde{w}_{i}}=\frac{\tilde{w}_{i}}{x} \\
& \left(\tilde{w}_{i}\right)_{x}=-\frac{\partial H}{\partial w_{i}}=-2 x\left(e^{2\left(w_{i+1}-w_{i}\right)}-e^{2\left(w_{i}-w_{i-1}\right)}\right),
\end{aligned}
$$

on the phase space $\mathbb{R}^{2\lfloor(n-1) / 2\rfloor+2}=\{(w, \tilde{w})\}\left(w=\left(w_{0}, \ldots, w_{\lfloor(n-1) / 2\rfloor}\right), \tilde{w}=\right.$ $\left.\left(\tilde{w}_{0}, \ldots, \tilde{w}_{\lfloor(n-1) / 2\rfloor}\right)\right)$ equipped with the symplectic structure

$$
\theta:=\sum_{i=0}^{\lfloor(n-1) / 2\rfloor} d w_{i} \wedge d \tilde{w}_{i}
$$

where the Hamiltonian $H$ is defined by

$$
H(w, \tilde{w} ; x):=\frac{1}{2 x} \sum_{i=0}^{\lfloor(n-1) / 2\rfloor} \tilde{w}_{i}^{2}-x \sum_{i=1}^{\lfloor(n-1) / 2\rfloor} e^{2\left(w_{i}-w_{i-1}\right)}-\frac{x}{2}\left(e^{-4 w_{\lfloor(n-1) / 2\rfloor}}+e^{4 w_{0}}\right) .
$$

The symplectic form $\theta$ is asymptotic to $\sum_{i=0}^{\lfloor(n-1) / 2\rfloor} d\left(\rho_{i} / 2\right) \wedge d\left(\gamma_{i} / 2\right)$ as $x \rightarrow 0$.

Remark 3. The Hamiltonian system may be written in terms of $X:=\log x$ as follows:

$$
\begin{aligned}
& H(w, \tilde{w} ; X):= \frac{1}{2 e^{X}} \sum_{i=0}^{\lfloor(n-1) / 2\rfloor} \tilde{w}_{i}^{2}-e^{X} \sum_{i=1}^{\lfloor(n-1) / 2\rfloor} e^{2\left(w_{i}-w_{i-1}\right)}-\frac{e^{X}}{2}\left(e^{-4 w_{\lfloor(n-1) / 2\rfloor}}+e^{4 w_{0}}\right) . \\
&\left(w_{i}\right)_{X}=\frac{\partial e^{X} H}{\partial \tilde{w}_{i}}=\tilde{w}_{i} \\
&\left(\tilde{w}_{i}\right)_{X}=-\frac{\partial e^{X} H}{\partial w_{i}}=-2 e^{2 X}\left(e^{2\left(w_{i+1}-w_{i}\right)}-e^{2\left(w_{i}-w_{i-1}\right)}\right) .
\end{aligned}
$$

\section{Symplectic structures and the Riemann-Hilbert Correspondence}

Both the asymptotic data $\gamma_{i}, \rho_{i}$ and the monodromy data $m_{i}, \log e_{i}^{\mathbb{R}}$ can be considered as defining local charts of the moduli space of solutions. From Theorem 1 we can show that the transformation between two charts via the Riemann-Hilbert correspondence is symplectic with respect to the "obvious" symplectic structure. The symplectic form $2 \theta$ we define in section 3 is asymptotic to the left hand side of the equality below as $x \rightarrow 0$.

\section{Theorem 4.}

$$
-\frac{1}{2} \sum_{i=0}^{\lfloor(n-1) / 2\rfloor} d \gamma_{i} \wedge d \rho_{i}=\sum_{i=0}^{\lfloor(n-1) / 2\rfloor} d m_{i} \wedge d \log e_{i}^{\mathbb{R}} .
$$

Remark 5. The left hand side is related to the Kirillov-Kostant form on a coadjoint orbit, and the right hand side is related to the Atiyah-Hitchin form on the space of the based rational maps of degree $n+1$ from $\mathbb{C} P^{1}$ to itself. Thus, both symplectic forms arise naturally from geometry. We shall present details of these facts elsewhere. 
Theorem 4 can be verified by direct calculation, but we prefer to give a proof by showing the existence of a generating function. The generating function will play an important role later.

Definition 6. Let

$$
\begin{aligned}
F\left(\rho_{0}, \ldots, \rho_{\lfloor(n-1) / 2\rfloor}, m_{0}, \ldots, m_{\lfloor(n-1) / 2\rfloor}\right): & =-\sum_{i=0}^{\lfloor(n-1) / 2\rfloor} \rho_{i} m_{i}+2 \log 2 \sum_{i=0}^{\lfloor(n-1) / 2\rfloor} m_{i}^{2} \\
& +\frac{n+1}{2} \sum_{k=0}^{n} \sum_{j=1}^{n} \psi^{(-2)}\left(\frac{m_{k-j}-m_{k}+j}{n+1}\right)
\end{aligned}
$$

where $m_{j+n+1}=m_{j}$ and $m_{j}=-m_{n-j}$. Here $\psi^{(-2)}(z)=\int_{0}^{z} \log \Gamma(x) d x=\frac{z(1-z)}{2}+$ $\frac{z}{2} \log 2 \pi+z \log \Gamma(z)-\log G(1+z)$, and $G$ is the Barnes G-function.

Theorem 7. The function $F$ is a generating function of the transformation

$\left(m_{0}, \ldots, m_{\lfloor(n-1) / 2\rfloor}, \rho_{0}, \ldots, \rho_{\lfloor(n-1) / 2\rfloor}\right) \mapsto\left(m_{0}, \ldots, m_{\lfloor(n-1) / 2\rfloor}, \log e_{0}^{\mathbb{R}}, \ldots, \log e_{\lfloor(n-1) / 2\rfloor}^{\mathbb{R}}\right)$

with respect to the given symplectic forms. More precisely, $F$ satisfies

$$
m_{i}=-\frac{\partial F}{\partial \rho_{i}}, \log e_{i}^{\mathbb{R}}=-\frac{\partial F}{\partial m_{i}} .
$$

Proof. The first identity is obvious. We show the second identity. Let

$$
\tilde{K}\left(m_{0}, \ldots, m_{n}\right):=(n+1) \sum_{i=0}^{n} \sum_{j=1}^{n} \psi^{(-2)}\left(\frac{m_{i}-m_{i+j}+j}{n+1}\right),
$$

where $m_{j+n+1}=m_{j}$. Let

$$
K\left(m_{0}, \ldots, m_{\lfloor(n-1) / 2\rfloor}\right):=\frac{1}{2} \tilde{K}\left(m_{0}, \ldots, m_{\lfloor(n-1) / 2\rfloor},-m_{\lfloor(n-1) / 2\rfloor}, \ldots,-m_{0}\right) .
$$

This $K$ is the last term of $F$ in (4.1). From the definition of $\log e_{i}^{\mathbb{R}}$ and $F$, it suffices to show that

$$
\begin{aligned}
& \frac{\partial K}{\partial m_{k}}\left(m_{0}, \ldots, m_{\lfloor(n-1) / 2\rfloor}\right) \\
& =\log \left(\frac{X_{k}\left(m_{0}, \ldots m_{\lfloor(n-1) / 2\rfloor},-m_{\lfloor(n-1) / 2\rfloor}, \ldots,-m_{0}\right)}{X_{n-k}\left(m_{0}, \ldots . m_{\lfloor(n-1) / 2\rfloor},-m_{\lfloor(n-1) / 2\rfloor}, \ldots,-m_{0}\right)}\right) .
\end{aligned}
$$

We can easily obtain that $X_{n-k}\left(-m_{n}, \ldots,-m_{0}\right)=\prod_{j=1}^{n} \Gamma\left(\frac{-m_{k}+m_{k-j}+j}{n+1}\right)$ and that $\tilde{K}\left(m_{0}, \ldots, m_{n}\right)=(n+1) \sum_{k=0}^{n} \sum_{j=1}^{n} \psi^{(-2)}\left(\frac{m_{k-j}-m_{k}+j}{n+1}\right)$. Then we obtain

$$
\frac{\partial \tilde{K}}{\partial m_{k}}=\log \left(X_{k}\left(m_{0}, \ldots, m_{n}\right) / X_{n-k}\left(-m_{n}, \ldots,-m_{0}\right)\right)
$$


Hence we have

$$
\begin{aligned}
& \frac{\partial K}{\partial m_{k}}\left(m_{0}, \ldots, m_{\lfloor(n-1) / 2\rfloor}\right) \\
= & \frac{1}{2}\left(\frac{\partial \tilde{K}}{\partial m_{k}}\left(m_{0}, \ldots, m_{\lfloor(n-1) / 2\rfloor},-m_{\lfloor(n-1) / 2\rfloor}, \ldots,-m_{0}\right)\right. \\
& \left.-\frac{\partial \tilde{K}}{\partial m_{n-k}}\left(m_{0}, \ldots, m_{\lfloor(n-1) / 2\rfloor},-m_{\lfloor(n-1) / 2\rfloor}, \ldots,-m_{0}\right)\right) \\
= & \frac{1}{2}\left(\log X_{k}\left(m_{0}, \ldots m_{\lfloor(n-1) / 2\rfloor},-m_{\lfloor(n-1) / 2\rfloor}, \ldots,-m_{0}\right)\right. \\
& -\log X_{n-k}\left(m_{0}, \ldots m_{\lfloor(n-1) / 2\rfloor},-m_{\lfloor(n-1) / 2\rfloor}, \ldots,-m_{0}\right) \\
& -\log X_{n-k}\left(m_{0}, \ldots m_{\lfloor(n-1) / 2\rfloor},-m_{\lfloor(n-1) / 2\rfloor}, \ldots,-m_{0}\right) \\
& \left.+\log X_{k}\left(m_{0}, \ldots . m_{\lfloor(n-1) / 2\rfloor},-m_{\lfloor(n-1) / 2\rfloor}, \ldots,-m_{0}\right)\right) \\
= & \log X_{k}\left(m_{0}, \ldots m_{\lfloor(n-1) / 2\rfloor},-m_{\lfloor(n-1) / 2\rfloor}, \ldots,-m_{0}\right) \\
& -\log X_{n-k}\left(m_{0}, \ldots . m_{\lfloor(n-1) / 2\rfloor},-m_{\lfloor(n-1) / 2\rfloor}, \ldots,-m_{0}\right) .
\end{aligned}
$$

This completes the proof.

\section{TAU FUnCTIONS AND THE CONSTANT PROBLEM}

In this section, we assume for simplicity that $n=3$, so $w=\left(w_{0}, w_{1}, w_{2}, w_{3}\right)$ with $w_{2}=-w_{1}, w_{3}=-w_{0}$. For general $n$ the same method applies. We consider only the global solutions, i.e., we assume $\log e_{i}^{\mathbb{R}}=0$, which means that the $\rho_{i}$ 's are determined by the $\gamma_{j}$ 's, as in Theorem 2. The following calculation is motivated by Theorem 1 in [11. See also [16] for further details.

Definition 8. Let us define the tau function of a global solution $w$ by

$$
\log \tau^{w}\left(x_{1}, x_{2}\right)=\int_{x_{1}}^{x_{2}} H\left(w_{i}(x), \tilde{w}_{i}(x), x\right) d x
$$

where $H$ is the Hamiltonian function.

Remark 9. Usually the tau function is defined (up to a multiplicative constant) by $\log \tau^{w}(x)=\int^{x} H\left(w_{i}(x), \tilde{w}_{i}(x), x\right) d x$. In that notation we have $\tau^{w}\left(x_{1}, x_{2}\right)=$ $\tau^{w}\left(x_{2}\right) / \tau^{w}\left(x_{1}\right)$.

The Hamiltonian function is

$$
H\left(x, w_{0}, w_{1}, \tilde{w}_{0}, \tilde{w}_{1}\right)=\frac{1}{2 x}\left(\tilde{w}_{0}^{2}+\tilde{w}_{1}^{2}\right)-x e^{2\left(w_{1}-w_{0}\right)}-\frac{x}{2}\left(e^{-4 w_{1}}+e^{4 w_{0}}\right)
$$

and is quasihomogeneous, that is,

$$
H(w, \lambda \tilde{w} ; \lambda x)=\lambda H(w, \tilde{w} ; x) \text { for any } \lambda>0 .
$$

It follows that

$$
\sum_{i=0}^{1} \tilde{w}_{i} \frac{\partial H}{\partial \tilde{w}_{i}}+x \frac{\partial H}{\partial x}=H
$$


For the solution $\left(w_{0}(x), w_{1}(x), \tilde{w}_{0}(x), \tilde{w}_{1}(x)\right)$ of (3.1) and (3.2), we have

$$
\begin{aligned}
\sum_{i=0}^{1} \tilde{w}_{i}(x) \frac{\partial H}{\partial \tilde{w}_{i}}\left(x, w_{0}(x), w_{1}(x), \tilde{w}_{0}(x), \tilde{w}_{1}(x)\right) & =\sum_{i=0}^{1} \tilde{w}_{i}(x)\left(w_{i}\right)_{x}(x) \\
x \frac{\partial H}{\partial x}\left(x, w_{0}(x), w_{1}(x), \tilde{w}_{0}(x), \tilde{w}_{1}(x)\right) & =-H\left(w_{0}(x), w_{1}(x), \tilde{w}_{0}(x), \tilde{w}_{1}(x)\right)+ \\
& \frac{d x H\left(x, w_{0}(x), w_{1}(x), \tilde{w}_{0}(x), \tilde{w}_{1}(x)\right)}{d x} .
\end{aligned}
$$

The first equality is obvious. The second equality follows from $\frac{d x H}{d x}=x \frac{d H}{d x}+H$ and

$$
\begin{aligned}
& \frac{d H\left(x, w_{0}(x), w_{1}(x), \tilde{w}_{0}(x), \tilde{w}_{1}(x)\right)}{d x} \\
& =\frac{\partial H}{\partial x}\left(x, w_{0}(x), w_{1}(x), \tilde{w}_{0}(x), \tilde{w}_{1}(x)\right)+\left(w_{i}\right)_{x}(x) \frac{\partial H}{\partial w_{i}}\left(x, w_{0}(x), w_{1}(x), \tilde{w}_{0}(x), \tilde{w}_{1}(x)\right) \\
& +\left(\tilde{w}_{i}\right)_{x}(x) \frac{\partial H}{\partial \tilde{w}_{i}}\left(x, w_{0}(x), w_{1}(x), \tilde{w}_{0}(x), \tilde{w}_{1}(x)\right) \\
& =\frac{\partial H}{\partial x}\left(x, w_{0}(x), w_{1}(x), \tilde{w}_{0}(x), \tilde{w}_{1}(x)\right) \\
& -\left(w_{i}\right)_{x}(x)\left(\tilde{w}_{i}\right)_{x}(x)+\left(\tilde{w}_{i}\right)_{x}(x)\left(w_{i}\right)_{x}(x) \\
& =\frac{\partial H}{\partial x}\left(x, w_{0}(x), w_{1}(x), \tilde{w}_{0}(x), \tilde{w}_{1}(x)\right) .
\end{aligned}
$$

Then it follows that

Proposition 10. $H=\tilde{w}_{0}\left(w_{0}\right)_{x}+\tilde{w}_{1}\left(w_{1}\right)_{x}-H+\frac{d}{d x}(x H)$.

Let $S\left(x_{1}, x_{2}\right):=\int_{x_{1}}^{x_{2}}\left(\sum_{i=0}^{1} \tilde{w}_{i}\left(w_{i}\right)_{x}-H\right) d x$, which is called the classical action, the functional from which we can derive the Euler-Lagrange equation using the fundamental lemma of calculus of variations. We obtain

$$
\begin{aligned}
\frac{\partial S\left(x_{1}, x_{2}\right)}{\partial \gamma_{j}} & =\int_{x_{1}}^{x_{2}}\left(\sum_{i=0}^{1}\left(\left(\tilde{w}_{i}\right)_{\gamma_{j}}\left(w_{i}\right)_{x}+\tilde{w}_{i}\left(\left(w_{i}\right)_{x}\right)_{\gamma_{j}}\right)-(H)_{\gamma_{j}}\right) d x \\
& =\int_{x_{1}}^{x_{2}}\left(\sum_{i=0}^{1}\left(\left(\tilde{w}_{i}\right)_{\gamma_{j}} \frac{\partial H}{\partial \tilde{w}_{i}}+\tilde{w}_{i}\left(\left(w_{i}\right)_{x}\right)_{\gamma_{j}}\right)-\sum_{i=0}^{1}\left(\frac{\partial H}{\partial w_{i}}\left(w_{i}\right)_{\gamma_{j}}+\frac{\partial H}{\partial \tilde{w}_{i}}\left(\tilde{w}_{i}\right)_{\gamma_{j}}\right)\right) d x \\
& =\int_{x_{1}}^{x_{2}}\left(\sum_{i=0}^{1}\left(\left(\tilde{w}_{i}\right)_{\gamma_{j}} \frac{\partial H}{\partial \tilde{w}_{i}}-\left(\tilde{w}_{i}\right)_{x}\left(w_{i}\right)_{\gamma_{j}}\right)-\sum_{i=0}^{1}\left(\frac{\partial H}{\partial w_{i}}\left(w_{i}\right)_{\gamma_{j}}+\frac{\partial H}{\partial \tilde{w}_{i}}\left(\tilde{w}_{i}\right)_{\gamma_{j}}\right)\right) d x \\
& +\left.\left(\sum_{i=0}^{1} \tilde{w}_{i}\left(w_{i}\right)_{\gamma_{j}}\right)\right|_{x_{1}} ^{x_{2}} \\
& =\left.\left(\tilde{w}_{0}\left(w_{0}\right)_{\gamma_{j}}+\tilde{w}_{1}\left(w_{1}\right)_{\gamma_{j}}\right)\right|_{x_{1}} ^{x_{2}} .
\end{aligned}
$$

The second equality follows from (3.1) and the chain rule, the third from integration by parts, and the fourth from (3.2). 
From the proposition and the definition of the $\tau$ function, we obtain

$$
\begin{aligned}
\frac{\partial}{\partial \gamma_{j}} \log \tau^{w}\left(x_{1}, x_{2}\right) & =\frac{\partial}{\partial \gamma_{j}} \int_{x_{1}}^{x_{2}}\left(\sum_{i=0}^{1} \tilde{w}_{i}\left(w_{i}\right)_{x}-H+\frac{d}{d x}(x H)\right) d x \\
& =\frac{\partial S\left(x_{1}, x_{2}\right)}{\partial \gamma_{j}}+\left(x_{2} H\left(x_{2}\right)-x_{1} H\left(x_{1}\right)\right)_{\gamma_{j}} \\
& =\left.\left(\sum_{i=0}^{1} \tilde{w}_{i}\left(w_{i}\right)_{\gamma_{j}}\right)\right|_{x_{1}} ^{x_{2}}+\left(x_{2} H\left(x_{2}\right)-x_{1} H\left(x_{1}\right)\right)_{\gamma_{j}} .
\end{aligned}
$$

At $x=0$ the form of (2.3) is

$$
w_{i}(x)=\frac{\gamma_{i}}{2} \log x+\frac{\rho_{i}}{2}+O\left(x^{\varepsilon_{i}}\right), \quad x \rightarrow 0
$$

for some $\varepsilon_{i}>0$ (which depends on $\gamma_{0}$ and $\gamma_{1}$ ); this can be shown as in Theorem 14.1 of [4] for the case $n=1$. This formula is differentiable in $x$ and the $\gamma_{i}$ 's. Therefore

$$
\begin{aligned}
\tilde{w}_{i} & =\frac{\gamma_{i}}{2}+O\left(x^{\varepsilon_{i}}\right), \\
\left(w_{i}\right)_{\gamma_{j}} & =\frac{\delta_{i, j}}{2} \log x+\frac{1}{2}\left(\rho_{i}\right)_{\gamma_{j}}+O\left(x^{\varepsilon_{i}} \log x\right), \\
\left(\tilde{w}_{i}\right)_{\gamma_{j}} & =\frac{\delta_{i, j}}{2}+O\left(x^{\varepsilon_{i}} \log x\right)
\end{aligned}
$$

as $x \rightarrow 0$.

At $x=\infty$, from [6], if $s_{1}^{\mathbb{R}} \neq 0$,

$$
w_{i}(x)=-s_{1}^{\mathbb{R}} 2^{-\frac{7}{4}}(\pi x)^{-\frac{1}{2}} e^{-2 \sqrt{2} x}+O\left(x^{-1} e^{-2 \sqrt{2} x}\right) \quad \text { as } x \rightarrow \infty,
$$

where $s_{1}^{\mathbb{R}}=-2 \cos \frac{\pi}{4}\left(\gamma_{0}+1\right)-2 \cos \frac{\pi}{4}\left(\gamma_{1}+3\right)$. If $s_{1}^{\mathbb{R}}=0$, we have

$$
\begin{aligned}
& w_{0}(x)=s_{2}^{\mathbb{R}} 2^{-\frac{5}{2}}(\pi x)^{-\frac{1}{2}} e^{-4 x}+O\left(x^{-1} e^{-4 x}\right) \sim O\left(x^{-1} e^{-2 \sqrt{2} x}\right) \\
& w_{1}(x)=-s_{2}^{\mathbb{R}} 2^{-\frac{5}{2}}(\pi x)^{-\frac{1}{2}} e^{-4 x}+O\left(x^{-1} e^{-4 x}\right) \sim O\left(x^{-1} e^{-2 \sqrt{2} x}\right),
\end{aligned}
$$

so the equation (5.2) holds for any generic $\left(\gamma_{0}, \gamma_{1}\right)$. The equation (5.2) is also differentiable in $x$ and the $\gamma_{i}$ 's, so

as $x \rightarrow \infty$.

$$
\begin{aligned}
& \tilde{w}_{i}(x)=s_{1}^{\mathbb{R}} 2^{-\frac{1}{4}} \sqrt{\pi} x^{\frac{1}{2}} e^{-2 \sqrt{2} x}+O\left(e^{-2 \sqrt{2} x}\right), \\
& \left(w_{i}\right)_{\gamma_{j}}=-\left(s_{1}^{\mathbb{R}}\right)_{\gamma_{j}} 2^{-\frac{7}{4}}(\pi x)^{-\frac{1}{2}} e^{-2 \sqrt{2} x}+O\left(x^{-1} e^{-2 \sqrt{2} x}\right), \\
& \left(\tilde{w}_{i}\right)_{\gamma_{j}}=\left(s_{1}^{\mathbb{R}}\right)_{\gamma_{j}} 2^{-\frac{1}{4}} \sqrt{\pi} x^{\frac{1}{2}} e^{-2 \sqrt{2} x}+O\left(e^{-2 \sqrt{2} x}\right)
\end{aligned}
$$

By substituting the above asymptotic expansions into (5.1) we obtain

$$
\frac{\partial}{\partial \gamma_{i}} \log \tau^{w}\left(x_{1}, x_{2}\right)=-\frac{\gamma_{i}}{4} \log x_{1}-\sum_{k=0}^{1} \frac{\gamma_{k}}{4}\left(\rho_{k}\right)_{\gamma_{i}}-\frac{\gamma_{i}}{4}+O\left(x_{1}^{\varepsilon_{i}} \log x_{1}\right)+O\left(x_{2}^{\frac{3}{2}} e^{-2 \sqrt{2} x_{2}}\right)
$$

as $x_{1} \rightarrow 0, x_{2} \rightarrow \infty$.

In our situation we have:

$$
\begin{gathered}
\tau^{w}(1, x)=C_{0} x^{\frac{1}{8}\left(\gamma_{0}^{2}+\gamma_{1}^{2}\right)}\left(1+O\left(x^{\varepsilon}\right)\right), \quad x \rightarrow 0, \\
\tau^{w}(1, x)=C_{\infty} e^{-x^{2}}\left(1+O\left(x^{1 / 2} e^{-2 \sqrt{2} x}\right)\right), \quad x \rightarrow \infty .
\end{gathered}
$$


Then

$$
\log \tau^{w}\left(x_{1}, x_{2}\right)=\log \frac{C_{\infty}}{C_{0}}-x_{2}^{2}-\frac{1}{8}\left(\gamma_{0}^{2}+\gamma_{1}^{2}\right) \log x_{1}+O\left(x_{1}^{\varepsilon}\right)+O\left(x_{2}^{1 / 2} e^{-2 \sqrt{2} x_{2}}\right) .
$$

Let

$$
C:=\log \frac{C_{\infty}}{C_{0}}=\lim _{\substack{x_{1} \rightarrow 0 \\ x_{2} \rightarrow \infty}}\left(\log \tau^{w}\left(x_{1}, x_{2}\right)+x_{2}^{2}+\frac{\gamma_{0}^{2}+\gamma_{1}^{2}}{8} \log x_{1}\right) .
$$

Then we obtain

$$
\frac{\partial C}{\partial \gamma_{i}}=\lim _{\substack{x_{1} \rightarrow 0 \\ x_{2} \rightarrow \infty}}\left(\frac{\partial}{\partial \gamma_{i}}\left(\log \tau^{w}\left(x_{1}, x_{2}\right)+x_{2}^{2}+\frac{\gamma_{0}^{2}+\gamma_{1}^{2}}{8} \log x_{1}\right)\right)=-\frac{\gamma_{i}}{4}-\sum_{k=0}^{1} \frac{\gamma_{k}}{4}\left(\rho_{k}\right)_{\gamma_{i}}
$$

that is,

$$
C=-\sum_{i=0}^{1} \frac{\gamma_{i}^{2}}{8}-\frac{1}{4} \sum_{k=0}^{1} \gamma_{k} \rho_{k}+\frac{1}{4} \int \sum_{k=0}^{1} \rho_{k} d \gamma_{k}
$$

Note that $\frac{\partial K}{\partial m_{i}}=-2 \frac{\partial K}{\partial \gamma_{i}}$, where $K$ is the function defined in the proof of theorem 7. and

$$
\int \sum_{k=0}^{1} \rho_{k} d \gamma_{k}=-(\log 2) \sum_{k=0}^{1} \gamma_{k}^{2}-2 K+\text { const. }
$$

The constant above is independent of the $\gamma_{i}$ 's. By substituting $\gamma_{0}=\gamma_{1}=0$, which corresponds to the trivial solution $w_{0} \equiv w_{1} \equiv 0$, into (5.3) , we obtain $C=$ $-4\left(\psi^{(-2)}(1 / 4)+\psi^{(-2)}(2 / 4)+\psi^{(-2)}(3 / 4)\right)+$ const. On the other hand, the tau function $\tau^{w}\left(x_{1}, x_{2}\right)$ corresponding to the trivial solution is $\exp \left(x_{1}^{2}-x_{2}^{2}\right)$, so $C=0$ in this case. In conclusion we have the following result:

\section{Theorem 11.}

$C=-\frac{1}{8}\left(\gamma_{0}^{2}+\gamma_{1}^{2}\right)-\frac{1}{2}\left(\gamma_{0} \rho_{0}+\gamma_{1} \rho_{1}\right)-\frac{1}{2} F+4\left(\psi^{(-2)}(1 / 4)+\psi^{(-2)}(2 / 4)+\psi^{(-2)}(3 / 4)\right)$.

The function $F$ in the theorem, which is the generating function, is given in Definition 6 .

\section{Appendix A. Monodromy DATA}

At $\zeta=0$ we have a formal solution $\Psi_{f}^{(0)}=e^{-w} \Omega\left(I+\sum_{i \geq 1} \Psi_{i}^{(0)} \zeta^{i}\right) e^{\frac{1}{\zeta} d_{n+1}}$ of (2.2), where

$$
d_{n+1}=\left(\begin{array}{cccc}
1 & & & \\
& \omega & & \\
& & \ddots & \\
& & & \omega^{n}
\end{array}\right), \Omega=\left(\begin{array}{ccccc}
1 & 1 & 1 & \cdots & 1 \\
1 & \omega & \omega^{2} & \cdots & \omega^{n} \\
1 & \omega^{2} & \omega^{4} & \cdots & \omega^{2 n} \\
\vdots & \vdots & \vdots & \ddots & \vdots \\
1 & \omega^{n} & \omega^{2 n} & \cdots & \omega^{n^{2}}
\end{array}\right) .
$$

We define the sector

$$
\Omega_{1}^{(0)}:= \begin{cases}\left(-\left(\frac{1}{n+1}+\frac{1}{2}\right) \pi, \frac{\pi}{2}\right) & (n+1 \in 2 \mathbb{Z}) \\ \left(-\left(\frac{1}{2(n+1)}+\frac{1}{2}\right) \pi,\left(\frac{1}{2(n+1)}+\frac{1}{2}\right) \pi\right) & (n+1 \in 2 \mathbb{Z}+1)\end{cases}
$$

where we use the notation $(a, b):=\left\{\zeta \in \mathbb{C}^{*} \mid a<\arg \zeta<b\right\}$. 
We let $\Omega_{k+\frac{1}{n+1}}^{(0)}=e^{-\frac{\pi}{n+1} \sqrt{-1}} \Omega_{k}^{(0)}\left(k \in \frac{1}{4} \mathbb{Z}\right)$ in the universal covering $\tilde{\mathbb{C}^{*}}$.

Let $\Psi_{k}^{(0)}$ be the fundamental solution such that $\Psi_{k}^{(0)} \sim \Psi_{f}^{(0)}$ on $\Omega_{k}^{(0)}$.

Similarly, at $\zeta=\infty$, we have the formal solution $\Psi_{f}^{(\infty)}=e^{w} \Omega^{-1}\left(I+\sum_{i \geq 1} \Psi_{i}^{(\infty)} \zeta^{-i}\right) e^{x^{2} \zeta d_{n+1}}$ and the sectors

$$
\begin{gathered}
\Omega_{1}^{(\infty)}:= \begin{cases}\left(-\frac{\pi}{2},\left(\frac{1}{n+1}+\frac{1}{2}\right) \pi\right) & (n+1 \in 2 \mathbb{Z}) \\
\left(-\left(\frac{1}{2(n+1)}+\frac{1}{2}\right) \pi,\left(\frac{1}{2(n+1)}+\frac{1}{2}\right) \pi\right) & (n+1 \in 2 \mathbb{Z}+1)\end{cases} \\
\Omega_{k+\frac{1}{n+1}}^{(\infty)}:=e^{\frac{\pi}{n+1} \sqrt{-1}} \Omega_{k}^{(\infty)} .
\end{gathered}
$$

Let $\Psi_{k}^{(\infty)}$ be the fundamental solution such that $\Psi_{k}^{(\infty)} \sim \Psi_{f}^{(\infty)}$ on $\Omega_{k}^{(\infty)}$.

We define the Stokes matrices $S_{k}^{(0)}, S_{k}^{(\infty)}$ by $\Psi_{k+1}^{(0)}=\Psi_{k}^{(0)} S_{k}^{(0)}, \Psi_{k+1}^{(\infty)}=\Psi_{k}^{(\infty)} S_{k}^{(\infty)}$.

We define the Stokes factors $Q_{k}^{(0)}, Q_{k}^{(\infty)}$ by $\Psi_{k+\frac{1}{n+1}}^{(0)}=\Psi_{k}^{(0)} Q_{k}^{(0)}, \Psi_{k+\frac{1}{n+1}}^{(\infty)}=$ $\Psi_{k}^{(\infty)} Q_{k}^{(\infty)}$.

Let $M:=Q_{1}^{(0)} Q_{1+\frac{1}{n+1}}^{(0)} \Pi$ where

$$
\Pi=\left(\begin{array}{cccc}
0 & 1 & & \\
& 0 & \ddots & \\
& & \ddots & 1 \\
1 & & & 0
\end{array}\right) .
$$

Let $m_{i}$ be the eigenvalues of $M$. It is proved in [6, 7] that the $m_{i}$ determine all $Q_{k}^{(0)}$.

We define the connection matrices $E_{k}$ by $\Psi_{k}^{(\infty)}=\Psi_{k}^{(0)} E_{k}$.

Let $E_{1}^{\text {global }}$ be $\frac{1}{n+1} A Q_{\frac{n}{n+1}}^{(\infty)}$ where

$$
A=\left(\begin{array}{lllll}
1 & & & \\
& & & 1 \\
& & . & \\
& 1 & &
\end{array}\right) .
$$

It is known that $E_{1}=E_{1}^{\text {global }}$ for global solutions $w$. Let $e_{i}^{\mathbb{R}}$ be the eigenvalues of $E:=E_{1}\left(E_{1}^{\text {global }}\right)^{-1}$. We have $e_{i}^{\mathbb{R}} e_{n-i}^{\mathbb{R}}=1$. It is proved in [7] that the $e_{i}^{\mathbb{R}}$ determine $E_{1}$.

\section{REFERENCES}

[1] P. P. Boalch, Quasi-Hamiltonian geometry of meromorphic connections, Duke Math. J. 139 (2007), no. 2, 369-405.

[2] S. Cecotti and C. Vafa, Topological-anti-topological fusion, Nuclear Phys. B 367 (1991), 359-461.

[3] B. Dubrovin, Geometry and integrability of topological-antitopological fusion, Comm. Math. Phys. 152 (1993), no. 3, 539-564.

[4] A. S. Fokas, A. R. Its, A. A. Kapaev and V. Y. Novokshenov, Painlevé transcendents: The Riemann-Hilbert Approach, Math. Surveys Monogr., 128, Amer. Math. Soc., Providence, RI, 2006.

[5] M. A. Guest, Topological-antitopological fusion and the quantum cohomology of Grassmannians, Jpn. J. Math. 16 (2021), no. 1, 155-183. 
[6] M. A. Guest, A. R. Its, and C.-S. Lin, Isomonodromy aspects of the tt* equations of Cecotti and Vafa II. Riemann-Hilbert problem, Comm. Math. Phys. 336 (2015), no. 1, 337-380.

[7] M. A. Guest, A. R. Its and C. S. Lin, Isomonodromy aspects of the tt* equations of Cecotti and Vafa III. Iwasawa factorization and asymptotics, Comm. Math. Phys. 374 (2020), no. 2, 923-973.

[8] N. Hitchin, Frobenius manifolds (notes by D. Calderbank), in [9].

[9] J. Hurtubise and F. Lalonde (eds.), Gauge theory and symplectic geometry, NATO ASI Series C: Maths \& Phys., 488, Kluwer, Dordrecht/Boston/London, 1995.

[10] A. Its, O. Lisovyy, Y. Tykhyy, Connection Problem for the Sine-Gordon/Painlevé III TauFunction and Irregular Conformal Blocks, Int. Math. Res. Not. IMRN, (2015), no. 18, 89038924.

[11] A. Its and A. Prokhorov, Connection problem for the tau-function of the sine-Gordon reduction of Painlevé-III equation via the Riemann-Hilbert approach, Int. Math. Res. Not. IMRN (2016), no. 22, 6856-6883.

[12] M. Jimbo, T. Miwa, K. Ueno, Monodromy preserving deformation of linear ordinary differential equations with rational coefficients I. General theory and $\tau$-function, Phys. D 2 (1981), no. 2, 306-352.

[13] B. M. McCoy, C. A. Tracy, and T. T. Wu, Painlevé functions of the third kind, J. Mathematical Phys. 18 (1977), no. 5, 1058-1092.

[14] T. Mochizuki, Harmonic bundles and Toda lattices with opposite sign, arXiv:1301.1718

[15] T. Mochizuki, Harmonic bundles and Toda lattices with opposite sign II, Comm. Math. Phys. 328 (2014), no. 3, 1159-1198.

[16] A. Prokhorov, Connection problem for Painlevé tau functions, Dissertation, Purdue University, 2019.

[17] L. Schlesinger, Über eine Klasse von Differentialsystemen beliebiger Ordnung mit festen kritischen Punkten, J. Reine Angew. Math. 141 (1912), 96-145.

[18] C. A. Tracy, Asymptotics of the $\tau$-function arising in the two-dimensional Ising model, Comm. Math. Phys. 142 (1991), 297-311.

[19] C. A. Tracy and H. Widom, Asymptotics of a class of solutions to the cylindrical Toda equations, Comm. Math. Phys. 190 (1998), 697-721.

Department of Pure and Applied Mathematics, Faculty of Science and Engineering, Waseda University, 3-4-1 Okubo, Shinjuku, Tokyo 169-8555 JAPAN

Email address: ryosuke.odoi@moegi.waseda.jp 